\title{
Simultaneous multiparticle emission from compound nuclei in evaporation process
}

\author{
Leonardo P. G. De Assis* \\ Centro Brasileiro de Pesquisas Físicas \\ Rua Dr. Xavier Sigaud 150, 22290-180 Rio de Janeiro-RJ, Brasil \\ E-mail: lpgassisecbpf.br
}

\section{Bianca M. Santos}

Instituto de Física, Universidade Federal Fluminense

Av. Gal. Milton Tavares de Souza, 24210-346 Niterói-RJ, Brasil

E-mail: biankceecbpf.br

\section{Sérgio B. Duarte}

Centro Brasileiro de Pesquisas Físicas

Rua Dr. Xavier Sigaud 150, 22290-180 Rio de Janeiro-RJ, Brasil

E-mail: sbdecbpf.br

\begin{abstract}
When a hot compound nucleus is formed as an intermediate state of a nuclear reaction, evaporation takes place. The conventional treatment for this process considers only single or sequential emission of particles. However, for very high excitation energy of the compound nucleus, other emission channels with several particles emitted simultaneously can be open. In the present work we introduce these channels in competition with the fission process in the decay chains of the compound hot nucleus formed in a spallation reaction. A Monte Carlo simulation is employed to determine the average yield of emitted particles along the decay chain. The neutron, proton, alpha and fission yields are obtained in the new version of the evaporation process and they are compared to yields of the conventional treatment with sequential single particle emission. The relevance of the different channels in competition is also shown.
\end{abstract}

XXXIV edition of the Brazilian Workshop on Nuclear Physics,

5-10 June 2011

Foz de Iguaçu, Parana state, Brasil

* Speaker.

$\dagger$ A footnote may follow. 


\section{INTRODUCTION}

Nuclear evaporation is the decay process usually employed for a hot intermediate compound nucleus formed in a nuclear reaction. For example, when high energy probes hit a heavy target, transferring a sufficiently high amount of energy, a spallation reaction takes place, involving the evaporation process. Presently great interest has been dedicated to this type of reaction, which is used to describe the neutron generation for the newest proposed generation of nuclear reactors, the Accelerator Driven System (ADS) [9]-[17]. The system is composed of a particle accelerator coupled to a reactor. The accelerated beam of charged particles collides with nuclei in the reactor medium, generating neutrons by spallation reactions. This neutron generation process works as an external source to induce fission in the reactor cycle. The main interest for this device arises due to the fact that it operates in a sub-critical regime, burning radioactive nuclear waste originated in conventional machines. However, as a relatively new proposal, and involving high energy beams, the ADS requires a deeper study, particularly concerning the spallation reaction.

When a charged particle bombards a target nucleus, a rapid cascade phase occurs with ejection of few nucleon leading to the formation of a hot residual nucleus. At this point the evaporation of particles begins through an evaporative decay chain. The fission reaction of the nucleus in the chain is considered in competition with the emission of neutrons, protons and alpha particles. For a typical beam energy of ADS system (around $1 \mathrm{GeV}$ for proton beam), the formed compound nucleus can reach an excitation energy of a few hundred $\mathrm{MeV}$, which is high enough to permit not only single emission, but also simultaneous particle evaporation. Such simultaneous emission channels should be investigated and constitute an open issue to be explored. The evaporation reaction chain includes many nuclei far from the beta stability line with no experimental data available. The nuclear properties of these nuclei are the object of intense theoretical and experimental physical research nowadays in order to understand the structure of these exotic nuclei. Consequently, in some calculations we have to deal with reasonable work in hypotheses or extrapolated results extracted from current model.

In the next section, the conventional and the multiparticle simultaneous evaporation are described. The results of our calculation with the implementation of simultaneous particle emission for the evaporation process using a Monte Carlo simulation is presented in section 3. We briefly present our conclusions in section 4 .

\section{EVAPORATION PROCESSES WITH SINGLE AND MULTIPLE SIMULTANEOUS EMISSION}

In a conventional treatment of nuclear evaporation, particles are emitted sequentially along the evaporative decay chain. The usual Weisskopf evaporation model [1] is the canonical prescription to determine the probabilities of single particle emission, given by $[1,12]$ :

$$
w_{k}=\frac{\Gamma_{k}}{\sum \Gamma_{i}}
$$


where

$$
\Gamma_{k}=\sigma \gamma_{k} \exp \left(2\left[\sqrt{a_{k} E_{k}^{*}}\right]-2\left[\sqrt{a E^{*}}\right]\right)
$$

The level density parameter for neutron emission is obtained by the Fermi gas approximation for a nuclear system, and reads,

$$
a_{n}=\tilde{a}\left\{1+\left[1-\exp \left(-0.05 E^{*}\right)\right] \frac{\Delta M}{E^{*}}\right\}
$$

where $\Delta M$ (in $M e V$ ) is the shell correction extracted from the mass formula [14] and

$$
\widetilde{a}=0.114 A+0.098 A^{\frac{2}{3}} \mathrm{MeV}^{-1}
$$

is a systematic phenomenological parametrization for the asymptotic value of $a_{n}$. A small correction due to the effect of the pairing energy is represented by additional terms in Eq (2.3). Here the level density for emission of other particles is given by,

$$
a_{k}=r_{k} a_{n}
$$

where $r_{k}$ is a dimensionless constant (used as $r=r_{p}=1$, according to Ref. [18] ). In Eq. (2.2), $\gamma_{k}$ is a constant related to the spin degenerecency factor given by,

$$
\gamma_{k}=\frac{g_{k} m_{k}}{\left(\pi^{2} h^{2}\right)}
$$

with $g_{k}=2$ (for neutrons and protons) and $g_{k}=1$ (for $\alpha$ particles); $m_{k}$ is the particle mass and $h$ Planck's constant and

$$
E_{k}^{*}=E^{*}-\bar{E}_{e v_{k}}
$$

where $E^{*}$ is the energy of the residual nucleus and $\bar{E}_{e v_{k}}$ is the average kinetic energy of the emitted particle.

The average total energy removed by the evaporation of a proton or alpha particle from cascade residual nucleus was calculated as [14]:

$$
\bar{E}_{e v_{k}}=\left(S_{k}+V_{k}\right)+2 \sqrt{\frac{E^{*}-\left(S_{k}+V_{k}\right)}{a_{k}}} .
$$

For neutrons we take $V_{k}=0$ in the above expression $S_{k}$ and $V_{k}$ are the separation energy and potential barrier, respectively.

We introduce multiple simultaneous emission in the evaporation chain as illustrated in Fig. 1. The first attempts to describe multiple simultaneous emissions were carried out by Tomosini [2], followed by a work of Beard [8]. 


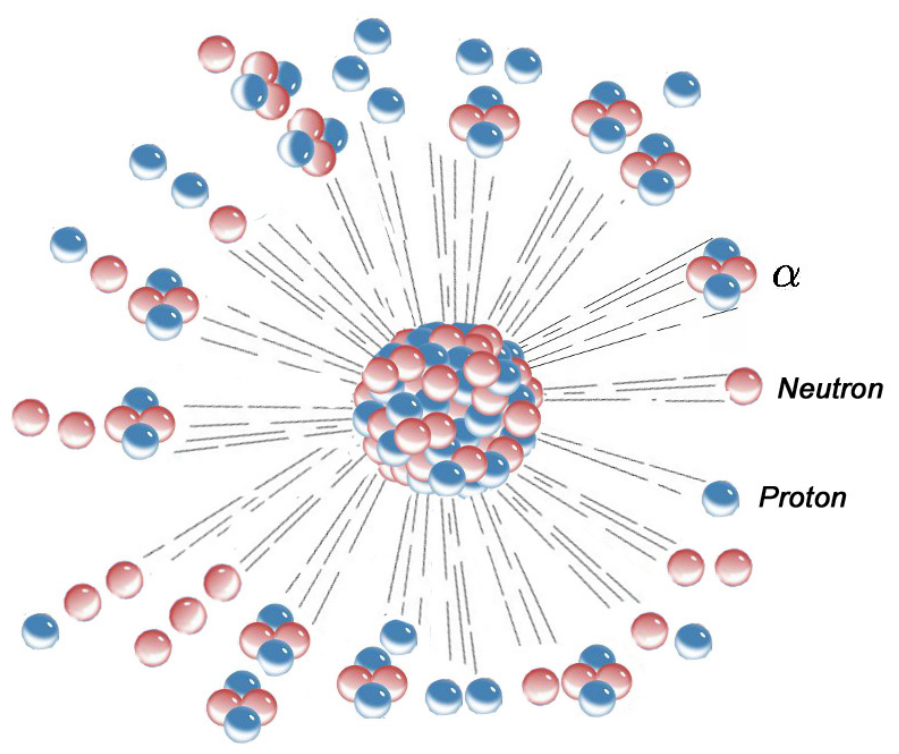

Figure 1: This figure show a pictoric representation of simultaneous multiparticle emission processes with all channels considered in this work.

They discussed the density of final states in phase space, without an direct application to a reaction process to determine the effect of the processes on the particles yields. As a first attempt to estimate this effect, we will consider that the probability of multiple simultaneous emission is determined by a Poisson like probability, determined as a product of the probabilities associated to the emission of each emitted particle.

$$
W=\prod_{k} w_{k}
$$

To determine the remaining excitation energy of the residual nucleus after each multiple emission we have to consider the energy carried away by the set of emitted particles, by evaluating the separation energies of the whole set of emitted particle and an approximate Coulomb potential barrier for the charged particles. The potential barrier for a charged particle (protons and alphas) in the i-th decay channel is taken as,

$$
V_{i}=C K_{i} \frac{Z_{i}\left(Z-Z_{i}\right) e^{2}}{r_{0}\left(A-A_{i}\right)^{1 / 3}+r_{0}^{j} A_{i}^{1 / 3}},
$$

where the subscript $i$ specifies the channel and the superscript $j$ denotes the reduced radius parameter associated to a set of outgoing particles. Namely, $r_{0}=1.2 \mathrm{fm}$ is the radius parameter for the residual nucleus; $r_{0}^{p}=1.14 \mathrm{fm}$ for $j$-channels involving protons without alphas and $r_{0}^{\alpha}=2.14 \mathrm{fm}$ for $j$-channels including at least one alpha among the emitted particle. 
The effective barrier penetrability factor, $K_{i}$, for the i-th channel with $n$-protons and $m$-alphas, is given by,

$$
K_{i}=K_{p}^{n} K_{\alpha}^{m}
$$

where $K_{p}$ and $K_{\alpha}$ are the proton and alpha barrier penetrability, respectively.

The separation energy of multiple particle emitted in the $\mathrm{i}$-channel is,

$$
S_{i}=m\left(Z-Z^{\prime}, A-A^{\prime}\right)+\sum_{i} m_{i}-m(Z, A)
$$

where $Z^{\prime}$ and $A^{\prime}$ are the total electric and baryonic charge, respectively, of emitted particles. The individual masses of these particles are the $m_{i}$.

In equation (2.10), $C=1-E^{*} / B\left(Z_{i}, A_{i}\right)$ represents the thermal correction to the chargedparticle Coulomb barrier [13] of the decaying nucleus with $B\left(Z_{i}, A_{i}\right)$ being its binding energy.

A Monte Carlo calculation is employed to simulate the decay chain by sampling the channel among the whole set of considered decay processes (single, multiple emissions and fission channels). When fission occurs the chain is ended. After each step of particle emission, the remaining excitation energy of the nucleus is determined and a new branch of the chain is created. When no energy is available for the subsequent decay, neither fission nor evaporation can take place, a spallation nuclear product is formed and the chain is terminated. The number of different emitted particles along the chain is accumulated to generate the particle yield. The number of times that the $i-t h$ channel is accessed generates the channel access number along the evaporation process.

\section{RESULTS}

We have run 600 thousand evaporative chain in our Monte Carlo evaluation of reaction observable. The illustrative target used was lead $\left({ }^{208} \mathrm{~Pb}\right.$ taken as the hot excited nucleus). We compare the total particle yield obtained with the calculation considering only sequential single emission, Fig.2, with the results of the evaporation including multiparticle emission Fig.3. In part (a) of these figures we can see that the neutron yield for the sequential single emission calculation presents a well located maximum around $150 \mathrm{MeV}$. The maximum number of neutrons is smaller for the case of single particle evaporation than the maximum obtained when multiparticle emission is considered. Also we can see a more steeply decreasing neutron yield, in the high energy region, in the case of single sequential emission. Comparing part-b of these figures, we note, in the high energy region, that the alpha particle yield is slightly greater than the proton yield, for the case of the single sequential emission evaporation chain, Fig. 2-b. This does not happen for the multiparticle simultaneous emission case. 

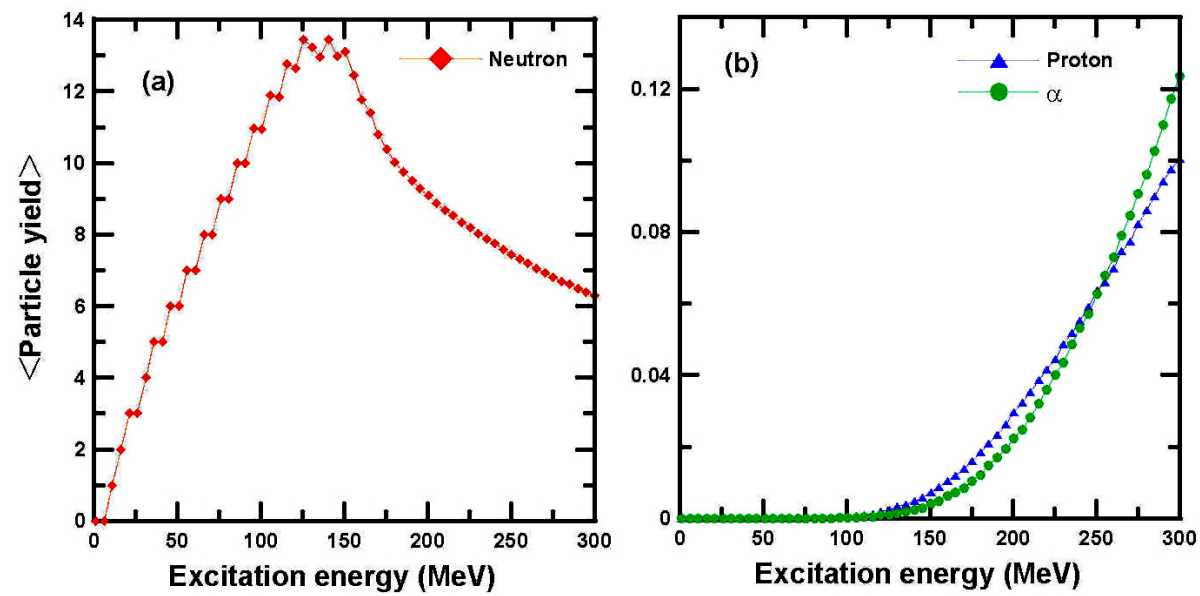

Figure 2: In part-a we show the neutron yield as a function of the residual nucleus excitation energy for the case of single sequential evaporative emission. In part-b the proton and alpha particle yields are displayed for the same calculation.
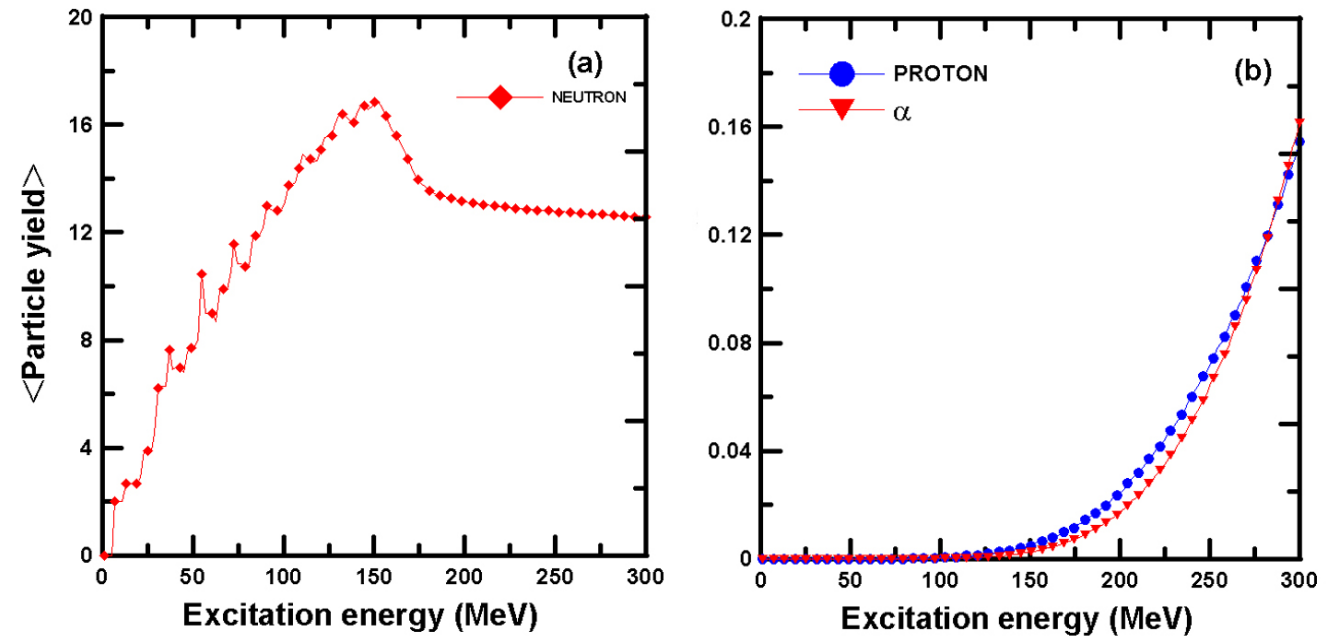

Figure 3: In these plots we show the particle yields for the case of the simultaneous multiparticle emission in the evaporative chain. In part-a the neutron yield is displayed as a function of the residual nucleus excitation energy, and in part-b the proton and alpha particle yield are shown. 

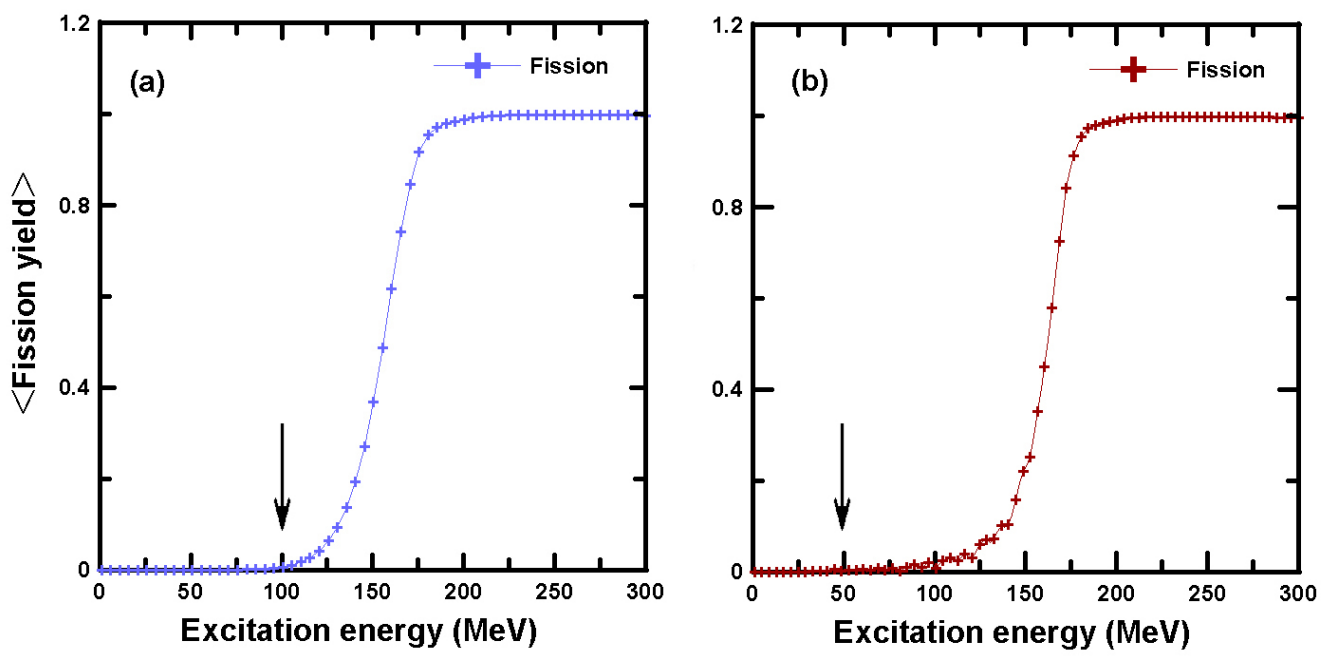

Figure 4: Yield of fission process for the case of single sequential emission calculation in (Fig.4-a) and for simultaneous multiparticle emission case, in part-b. The arrow indicate the energy when the fission yield begins to increase.

In Fig. 4 we present we compare the total yield of fission processes for the calculations carried out. Note the similar behaviour for both results, however with the curve for multiparticle simultaneous emission in part-b shifted (by almost $50 \mathrm{MeV}$ ) to smaller values of the residual nucleus excitation energy, when compared with the curve for single sequential in part-a.

The average number of accesses to a given channel of multiparticle emission along the Monte Carlo calculation is shown in Fig. 5. These curves show the relevance of the considered channels to the calculation. They are grouped by similar magnitude of the average number of accesses. The more relevant ones are those with pure neutron emission, single, double and ternary, and fission processes, grouped in Fig 5(a). In the sequence, single proton and alpha emission are grouped in part-b. The binary and ternary simultaneous emissions are present in part-c and part-d. The less significant channels regarding the access number are the ternary emission including charged particles in part-d. 

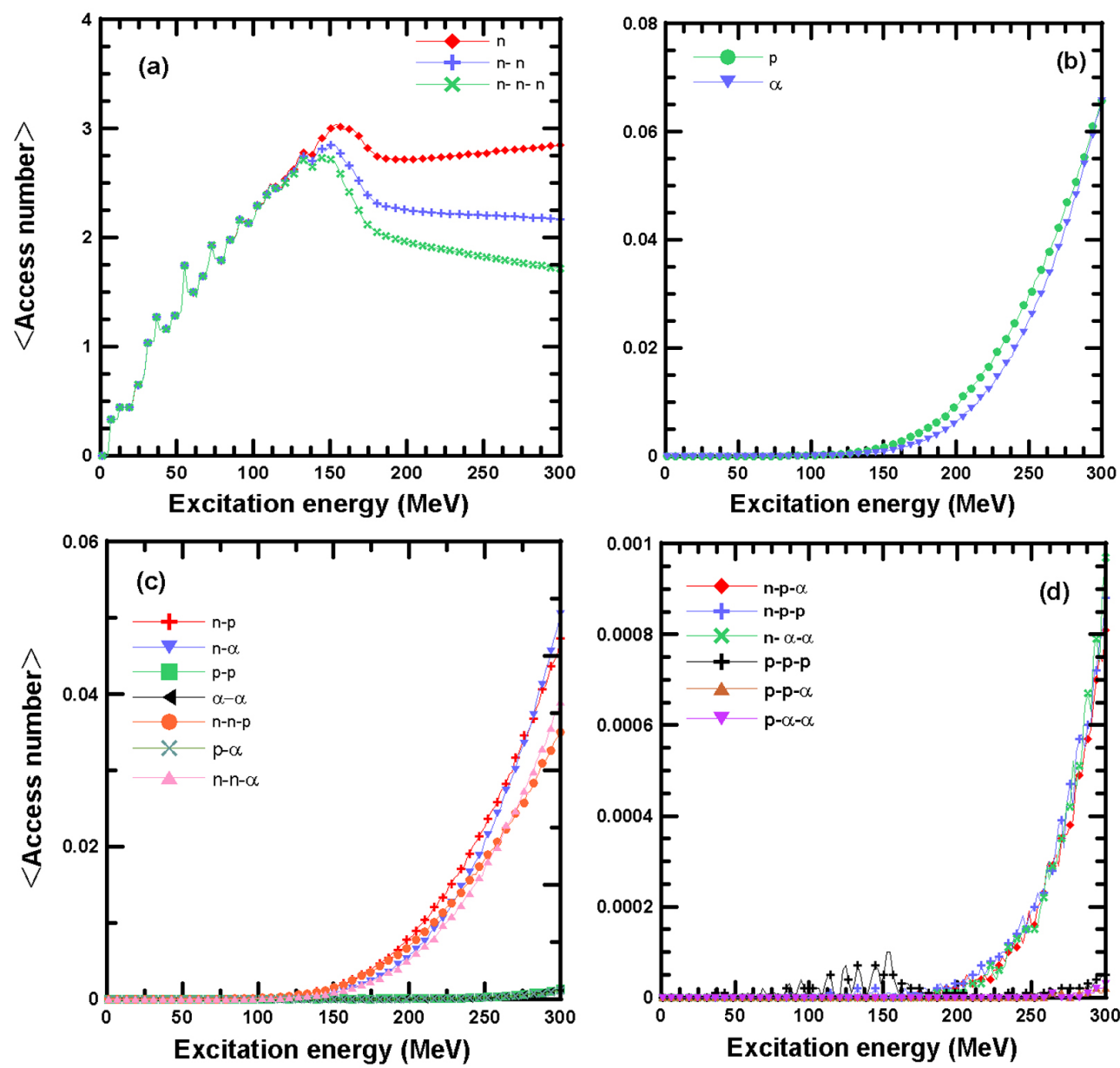

Figure 5: Average number of accesses to a given channel as a function of the excitation energy of the residual nucleus, for the case of the simultaneous multiparticle evaporation calculation. The channel are grouped according to the magnitude of the average access number, showing the probabilistic relevance of the different channels considered in the calculation.

\section{CONCLUSION}

The evaporation of a hot compound nucleus is revisited by considering the inclusion of simultaneous multiple particle emission channels. The relevance of these processes is shown for the case a heavy compound nucleus formed with excitation energy in the range of few $\mathrm{MeV}$ to three hundred $\mathrm{MeV}$. The average particle yield is determined and compared to the case of the conventional calculation. The average number of accesses to the different channels considered in the evaporation chain is also shown.

We call attention to the fact that here the definition of the probabilities associated with the multiple particle emission channels was only a reasonable attempt to carrier out the calculation. In spite of this the results are consistent with what is expected for the situation of increasing excitation energy of the compound nucleus. A calculation using probabilities based on the phase space density 
of the particle final state are in progress.

\section{References}

[1] V. F. Weisskopf, Statistics and Nuclear Reaction, Physical Review, 52, 295-303, 1937.

[2] A. Tomasini, Multiple Emissions in Nuclear Reactions, Il Nuono Cimento, VI, 2 2264, 1957.

[3] E. Fermi, High Energy Nuclear Events, Prog. Theo. Physics, 5, 570, 1950.

[4] I. L. Rozental, The Fermi Theory of Multiple Particle Production in Nucleon Encounters, Sov. Phys. JETP, 28, 118, 1955.

[5] E. Makowska, Fermi Model Description of High Multiplicity Compound Nucleus decay, Acta Physica Polonica, bf B10, 11, 997, 1979.

[6] J. V. Lepore, R. N. Stuart, Nuclear Events at High Energies, Physical Review, 94, 6, 1724, 1954.

[7] T. Ericson, A New Technique in the Statistical Model of Particle Production, Il Nuono Cimento, XXI, $4605,1961$.

[8] D. B. Beard, A. McLellan, Quantitative Nuclear Evaporation Theory and the Nuclear Potential, Physical Review, 131, 6, 2664, 1963.

[9] C. Rubbia,J. A. Rubio, S. Buorno, F. Carminati, N. Fiétier,L. Calvez, C. Geles, Y. Kadí, R. Klapisch, P. Mandrillon, J. P. Revol, C. H. Roche, Conceptual Design of a Fast Neutron Operated High Power Energy Amplifier. CERN/AT/95-44(ET) 1995.

[10] H. Nifenecker, O. Meplan, S. David, Accelerator Driven Subcritical Reactos, Institute of Physics Publishing Bristol and Philadelphia, 2003.

[11] P. Saracco, G. Ricco, Various Operating Regimes of a Subcritical System as a function of Subcriticality in One-Group Theory, Nuclear Science Engineering, 162, 167-177, 2009.

[12] Bianca M. Santos, Análise Neutrônica de Reatores com FonteE Estimulada por Feixe de Prótons em "Accelerator-Driven Systems - ADS", Master Thesis-CBPF, 2011.

[13] O.A.P. Tavares, M.L. Terranova, Z. Phys. A: Hadr. Nucl. 343 , 407,1992.

[14] O.A.P. Tavares, E.L. Medeiros, Level density parameter at the fission point from intermediate-energy photofission cross section data. Journal of Physics G: Nuclear and Particle Physics, 30, 395-405, 2004.

[15] A. Deppman, O. A. P. Tavares, S. B. Duarte, E. C. Oliveira, J. D. T. Arrua-Neto, S. R. Pina, V .P. Likhachev, O. Rodriguez, J. Mesa, M. Gonçalves, The MCEF code for nuclear evaporation and fission calculations, Computer Physics Communication, 145, 385-394, 2002.

[16] A. Deppman,; O. A. P. Tavares, S. B. Duarte, J. D. T. Arrua-Neto, M. Gonçalves, V .P. Likhachev, J. Mesa, E. C. Oliveira, S. R. Pina, O. Rodriguez, A Monte Carlo method for nuclear evaporation and fission at intermediate energies, Nuclear Instruments and Methods in Physics Research B, 211, 15-21, 2003.

[17] S. Anefalos, A. Deppman, G. Silva, J. R. Maiorino, A. Santos, S. B. Duarte, O. A. P. Tavares, Spallation Product Distributions and Neutron Multiplicities for Accelerator-Driven Systems Using the CRISP Code, Nuclear Science and Engineering, 159, 102-105, 2008.

[18] K.J. LeCouteur, Spin Orbit Coupling in the Nuclear Shell Model, Proc. Phys. Soc. London A, 63, 259,1950 . 